Article

\title{
Reduced Glutamine Synthetase Activity Alters the Fecundity of Female Bactrocera dorsalis (Hendel)
}

\author{
Dong Wei ${ }^{1,2} \oplus$, Meng-Yi Zhang ${ }^{1,2}$, Ying-Xin Zhang ${ }^{1,2}$, Su-Yun Zhang ${ }^{1,2}$, Guy Smagghe ${ }^{1,2,3}$ \\ and Jin-Jun Wang ${ }^{1,2, *}$ \\ 1 Chongqing Key Laboratory of Entomology and Pest Control Engineering, College of Plant Protection, \\ Southwest University, Chongqing 400716, China \\ 2 International Joint Laboratory of China-Belgium on Sustainable Crop Pest Control, State Cultivation Base of \\ Crop Stress Biology for Southern Mountainous Land, Academy of Agricultural Sciences, Southwest \\ University, Chongqing 400716, China \\ 3 Department of Plants and Crops, Faculty of Bioscience Engineering, Ghent University, Ghent 9000, Belgium \\ * Correspondence: wangjinjun@swu.edu.cn; Tel.: +86-23-6825-0255
}

Received: 4 May 2019; Accepted: 25 June 2019; Published: 27 June 2019

check for updates

\begin{abstract}
Glutamine synthetase (GS) is a key enzyme in glutamine synthesis and is associated with multiple physiological processes in insects, such as embryonic development, heat shock response, and fecundity regulation. However, little is known about the influence of GS on female fecundity in the oriental fruit fly, Bactrocera dorsalis. Based on the cloning of $B d G S s$, mitochondrial BdGSm and cytoplasmic $B d G S c$, we determined their expressions in the tissues of adult $B$. dorsalis. BdGSm was highly expressed in the fat body, while $B d G S c$ was highly expressed in the head and midgut. Gene silencing by RNA interference against two $B d G S s$ isoforms suppressed target gene expression at the transcriptional level, leading to a reduced ovarian size and lower egg production. The specific inhibitor L-methionine S-sulfoximine suppressed enzyme activity, but only the gene expression of $B d G S m$ was suppressed. A similar phenotype of delayed ovarian development occurred in the inhibitor bioassay. Significantly lower expression of vitellogenin and vitellogenin receptor was observed when GS enzyme activity was suppressed. These data illustrate the effects of two GS genes on adult fecundity by regulating vitellogenin synthesis in different ways.
\end{abstract}

Keywords: oriental fruit fly; glutamine synthetase; fecundity; L-methionine S-sulfoximine; vitellogenin

\section{Introduction}

Glutamine synthetase (GS, EC 6.3.1.2, L-glutamate: ammonia ligase) is widely distributed in microorganisms, animals, and higher plants, and is involved in many biological processes. It is required to modulate the level of the neurotransmitter glutamate and the level of glutamine biosynthesis. GS is also an essential detoxification enzyme in stress and immune responses [1,2]. Recently, a new role for GS in endothelial cell migration during pathological angiogenesis has been reported in mammals, beyond that of glutamine synthesis [3]. GS is classified into three distinct form: GSI, which is present only in prokaryotes; GSII, found in eukaryotes and some prokaryotes; and GSIII, which is present in anaerobes [4]. In insects, the two types of GS isoforms are mitochondrial GS and cytoplasmic GS, both belonging to the GSII form [5].

The physiological function of both GS isoforms has seldom been reported in insects. In Drosophila, mitochondrial GS is necessary in the early stages of embryonic development, and interruption of GS expression causes female sterility [6,7]. In Aedes aegypti, GS is continuously expressed at all developmental stages [8], and provides the glutamine needed for the initial steps of chitin biosynthesis 
in the female peritrophic matrix [9]. In Apis cerana cerana, GS is involved in response to environmental stress, e.g., thermal stress, oxidative stress, and pesticides [2]. In Acyrthosiphon pisum, GS works with glutamate synthase (Glts) to incorporate ammonium nitrogen into glutamate. This is a key source of nitrogen, fueling integrated amino acid metabolism in the aphid-Buchnera endosymbiont partnership [10]. GS is also an important regulator involved in female Nilaparvata lugens fecundity by activating the target of the rapamycin signal pathway [11,12]. The microRNA miR-4868b also plays a crucial role in targeting the GS gene in N. lugens [11].

The oriental fruit fly, Bactrocera dorsalis (Hendel), is one of the most economically important fruit flies. Its high reproductive rate can lead to significant crop losses $[13,14]$. Two type II BdGSs genes, mitochondrial BdGSm and cytoplasmic BdGSc, have been cloned from B. dorsalis, and the function of $B d G S c$ in larval development has been studied [15]. Their high expression in adults indicates an important role in the adult stage. In this study, we provide experimental evidences demonstrating that $B d G S m$ and $B d G S c$ are functionally involved in B. dorsalis fecundity.

\section{Materials and Methods}

\subsection{Insects}

A stock colony of B. dorsalis was collected as pupae from Haikou, Hainan Province of China, in 2008 , and then continuously maintained in our laboratory. The larvae and adults were cultured on an artificial diet at $27.5 \pm 0.5{ }^{\circ} \mathrm{C}$, relative humidity of $75 \pm 5 \%$, and $14 \mathrm{~h}$ light: $10 \mathrm{~h}$ dark photoperiod [16]. All insects used for experiments were of the same age.

\subsection{Gene Expression in Adults}

Both $B d G S$ s genes, $B d G S m$ and $B d G S c$, are expressed at high levels in adult $B$. dorsalis [15]. In this study, we detected their expressions during maturation of the adult stage. Newly emerged adults were caged, and female and male adults were maintained separately. Virgin female and male adults at 0 to $10 \mathrm{~d}$ were collected for total RNA isolation using TRIzol reagent (Invitrogen, Carlsbad, CA, USA) according to manufacturer's instructions. The quality was evaluated by a NanoDrop One instrument (Thermo Fisher Scientific, Madison, WI, USA). After genomic DNA digestion with RQ1 RNase-free DNase (Promega, Madison, WI, USA), the RNA samples were used for first-strand complementary DNA synthesis using a PrimeScript kit (Takara, Dalian, China). The relative expression of both BdGSs genes was calculated by a quantitative real-time polymerase chain reaction (qRT-PCR) protocol as used in previous studies $[17,18]$, with a CFX384 Optics Module (Bio-Rad, Singapore). The qRT-PCR reaction was carried out in a $10 \mu \mathrm{L}$ reaction volume including $5 \mu \mathrm{L}$ of Novostar-SYBR Supermix (Novoprotein, Shanghai, China), $3.5 \mu \mathrm{L}$ of nuclease-free water, $0.5 \mu \mathrm{L}$ of cDNA ( $400-500 \mathrm{ng} / \mu \mathrm{L})$, and $0.5 \mu \mathrm{L}$ each of forward and reverse primers $(10 \mu \mathrm{M})$. A melting curve analysis from 60 to $95{ }^{\circ} \mathrm{C}$ was conducted at the end to ensure the specificity and consistency of all generated products. The fragments of 185 and $150 \mathrm{bp}$ from BdGSm and BdGSc were selected for qRT-PCR [15]. Alpha-tubulin was used as an internal reference gene [19]. Thereafter, the head, thorax, midgut, fat body, Malpighian tubules, and ovary tissues from 9-day-old virgin female adults were dissected in phosphate-buffered solution (PBS, $\mathrm{pH}=7.2$ ) for total RNA isolation as above. The relative expressions of both $B d G S s$ genes among the adult tissues were also analyzed by qRT-PCR. The gene expression in adults post-eclosion and gene expression among tissues were conducted with three biological replicates, and analyzed by qBase Plus software (Biogazelle, Ghent, Belgium) [20].

\subsection{RNAi Bioassay}

The specific fragments of 497 and $345 \mathrm{bp}$ from the open reading frame of BdGSm and BdGSc were selected and amplified, respectively. The primers of $B d G S c$ were the same as in a previous study [15], and the primers of BdGSm were as follows: forward $\left(5^{\prime} \rightarrow 3^{\prime}\right)$ taatacgactcactataggg GAACCTTGGTTCGGCATTG, reverse $\left(5^{\prime} \rightarrow 3^{\prime}\right)$ taatacgactcactataggg CCTACGATCCAAAGGAAGG. 
These fragments show a short overlap with the region for qRT-PCR. Both BdGSs gene-specific double-stranded RNAs were synthesized using the Transcript Aid T7 High Yield Transcription Kit (Thermo Scientific, Vilnius, Lithuania). Three batches of females (30 individuals in each) were injected with gene-specific dsRNA of both BdGSs genes at 2-, 4-, and 6-day-old three times with $2 \mu \mathrm{g}$ dsRNA each. Females injected with the same amount of dsGFP were used as a negative control. One batch of injected females was used for total RNA isolation and cDNA preparation as above. The RNAi efficiencies were determined and calculated by qRT-PCR with three biological replicates. The gene expression between control and treatments were analyzed using the $2^{-\Delta \Delta \mathrm{Ct}}$ method [21]. Twenty of the second batch of injected females were dissected in PBS. The ovaries were observed under a binocular stereoscope (KEYENCE, VHX-S550E, Osaka, Japan), and the sizes of each pair of ovaries were calculated according to the mean diameter of each ovary. Thirty of the third batch of injected females were exposed to males of the same age for mating. Ten mated females were randomly selected and separately reared for egg production. Subsequent egg-laying was recorded using a previously described method [14]. Briefly, the orange juice in pored Eppendorf tube was used as the attractant in the container, and the number of eggs was recorded every day. The difference of the RNAi efficiency and the mean number of eggs laid per female individual was analyzed using a Student's $t$-test $(P<0.05)$ and SPSS 19.0 (IBM, Chicago, IL, USA).

\subsection{The MSX Bioassay}

The GS-specific inhibitor, L-methionine S-sulfoximine (MSX, Sigma, Shanghai, China), which irreversibly blocks the catalytic activity of GS [12], was used to observe the gene expression and enzymatic activity in $B$. dorsalis adult females. Three groups (30 individuals in each) of newly emerged adult females were reared on an artificial diet for two days and then reared with $10 \mu \mathrm{L} /$ fly of MSX-added solution at three doses of $0.5,1.0$, and $2.5 \mu \mathrm{M}$ every day. Flies fed on a diet with only purified water added were used as the control. One group of the test female flies was collected after five days of MSX feeding (8-day-old) for gene expression and enzymatic activity determination. The total RNA isolation and RT-qPCR were conducted and analyzed as above to study the gene expression. Enzymatic activity was determined using the GS enzyme reagent kit (Solarbio, Beijing, China) according to manufacturer's instructions and the methods as used in a previous study [15]. Thirty of the second group of females were collected for ovary dissection in PBS. Ovary diameter was the parameter used to evaluate female ovarian size as described above. The third group of females was collected and allowed to mate with males of the same age. Mating successes were recorded, and the fecundity of the mated females was calculated based on the number of eggs laid for at least five days. The analysis of the ovarian size was performed in the same manner as in the RNAi bioassay. The expressions of two marker genes, vitellogenin (BdVg1, AF368053) and vitellogenin receptor (BdVgR, JX469118) [18,22], were thereafter detected by qRT-PCR. Three biological replicates were performed.

\subsection{Statistical Analysis}

The significant differences of both $B d G S s$ genes expressions among tissues were evaluated by one-way analysis of variance (ANOVA) with a Turkey LSD using SPSS 19.0 (IBM, Chicago, IL, USA) $(P<0.05)$. The gene expression and enzymatic activity of females feeding with MSX were also analyzed by one-way ANOVA. The significant differences of the RNAi efficiency, the diameter of the ovaries, the mean number of eggs laid per female individual, and two marker genes' expressions were analyzed using Student's $t$-test $(P<0.05)$.

\section{Results}

\subsection{Gene Expression during Sex Maturation}

The gene expression data showed that both BdGSs genes were widely expressed in the adult stages (Figure 1). We then determined their expressions in various tissues of 9-day-old female adults. 
Relative expression profiling showed that $B d G S m$ was prominently expressed in the fat body of female adults, while $B d G S c$ was highly expressed in most of the tested tissues except for the reproductive tissue (Figure 2). The difference in expression may indicate different roles in B. dorsalis female adults.
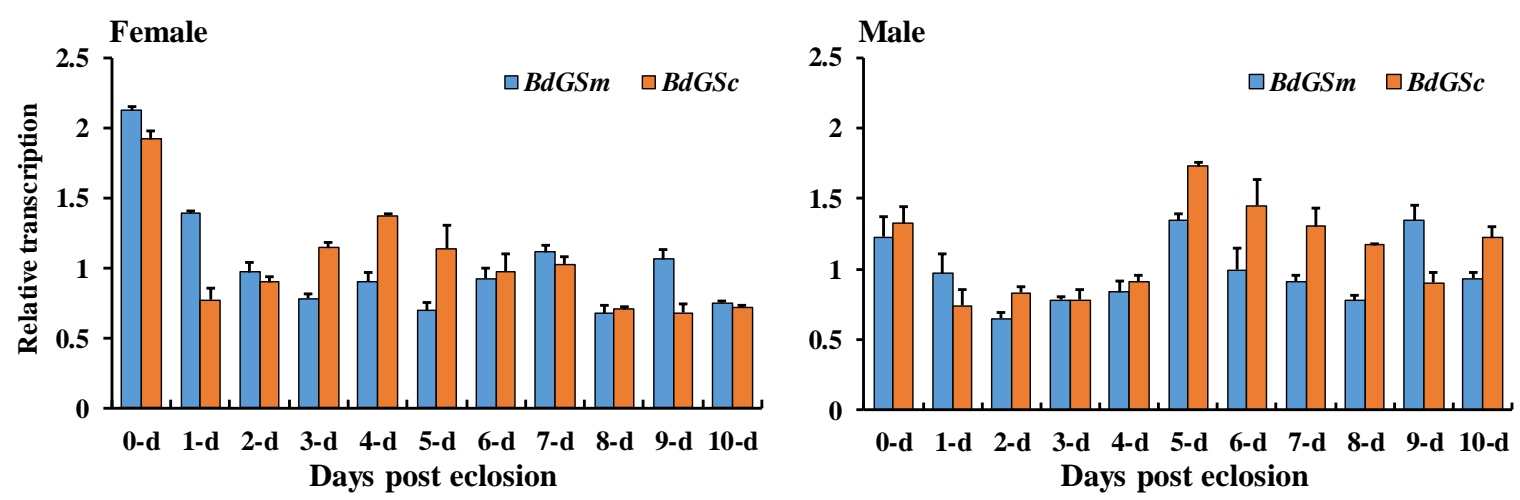

Figure 1. Gene expression of $B d G S m$ and $B d G S c$ during female and male adult post-eclosion. The gene expression was calculated by qBase Plus software. The bar represents the mean gene expression and the error bar represents the positive standard error of the mean.
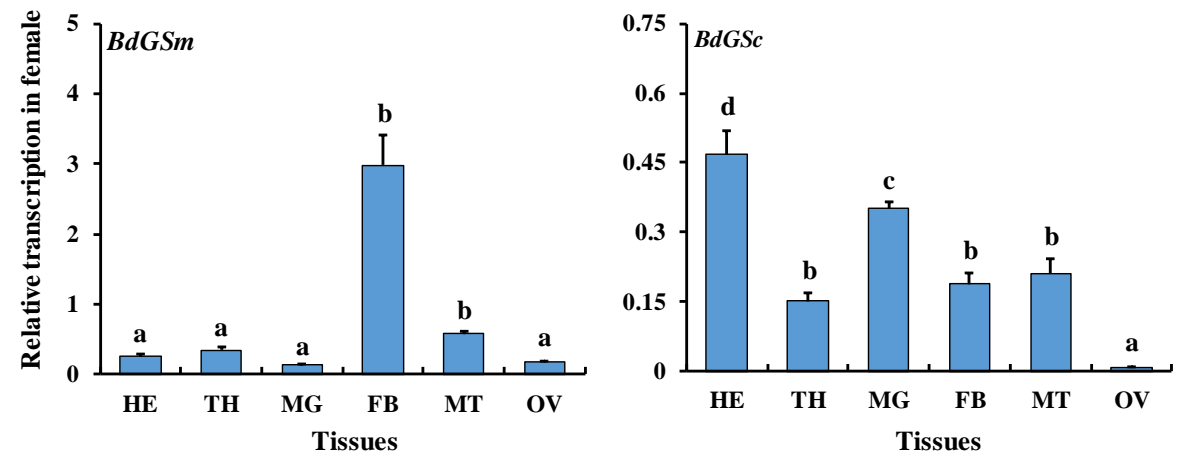

Figure 2. Gene expression of $B d G S m$ and $B d G S c$ among tissues from 9-day-old female adults. HE, TH, MG, FB, MT, and OV stand for head, thorax, midgut, fat body, Malpighian tubules and ovary, respectively. The gene expression was calculated by qBase Plus software. The bar represents the mean gene expression and the error bar represents the positive standard error of the mean. A different letter on the bar indicates a significant difference analyzed by ANOVA with a Tukey LSD test using SPSS 19.0 $(P<0.05)$.

\subsection{RNAi Bioassay}

We used RNAi to investigate the potential roles of $B d G S m$ and $B d G S c$ in the female fecundity of $B$. dorsalis. The results indicated that both $B d G S$ s genes can be suppressed by gene-specific double-stranded RNA injection. The silencing efficiencies of $B d G S m$ and $B d G S c$ were $75.4 \%$ and $71.8 \%$, respectively, in $B$. dorsalis adults (Figure $3 \mathrm{~A}$ ), after three serial injections with dsRNA at 2-, 4-, and 6-day-old. To investigate their roles in female fecundity, the GS-suppressed females were dissected for ovarian size determination. The average ovarian size of the residual females was smaller in the dsRNA-BdGSs group than in the control group $(P=0.015$, Figure $3 B)$. The frequency of ovarian size differed between categories. In the control, $60 \%$ of the ovaries were $>1.3 \mathrm{~mm}$, while $30 \%$ were $>1.3 \mathrm{~mm}$ in the BdGSm-silenced group, and only $15 \%$ were $>1.3 \mathrm{~mm}$ in the BdGSc-silenced group (Figure 3C). It appears that both $B d G S$ s genes are involved in the ovarian development of $B$. dorsalis females. 

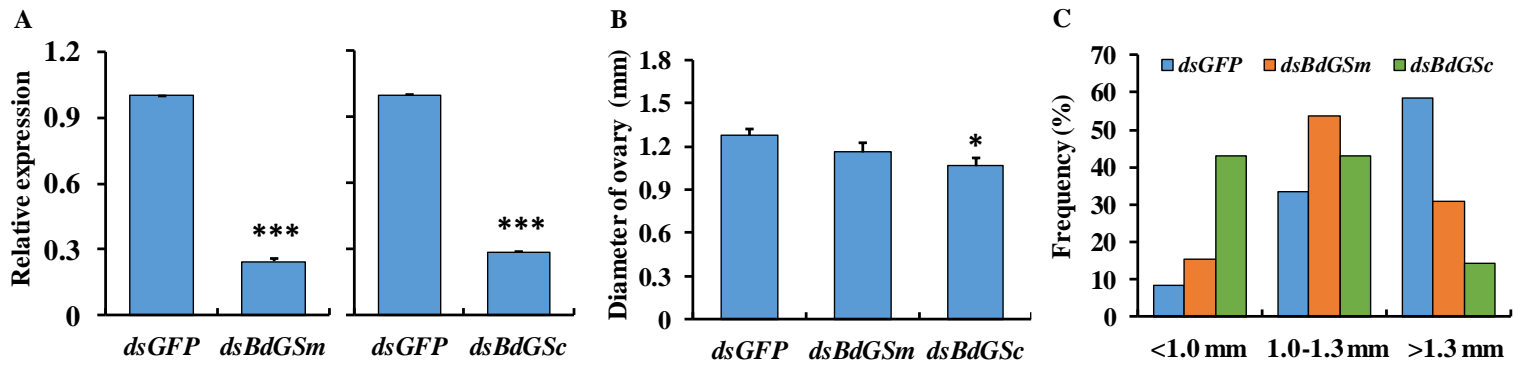

Figure 3. The silencing efficiency for the two BdGSs (A), ovarian diameter (B), and the frequency of the ovarian size (C) after three subsequent double-stranded RNA injections. The gene expression in the control group was calculated and normalized to be 1 . The bar represents the mean gene expression, the mean ovarian diameter, and the frequency of ovarian size category $(n=20)$. The error bar represents the positive standard error of the mean. The asterisk above the bar indicates the significant difference compared with the control as analyzed by Student's $t$-test using SPSS $19.0\left({ }^{*} P<0.05 ;{ }^{* * *} P<0.001\right)$. The bars in panel $\mathrm{C}$ represent the frequency of ovarian size of each category.

Females injected with dsRNA three times were mated with wild males and the eggs were counted for five days. There was no difference in mating success between any target gene silencing treatment and the control. The daily number of eggs in the silenced treatments was less than that in the control, but the daily counts were similar. However, the total number of the eggs over the five days of oviposition was significantly less in the silenced treatments than in the control group (Figure 4).

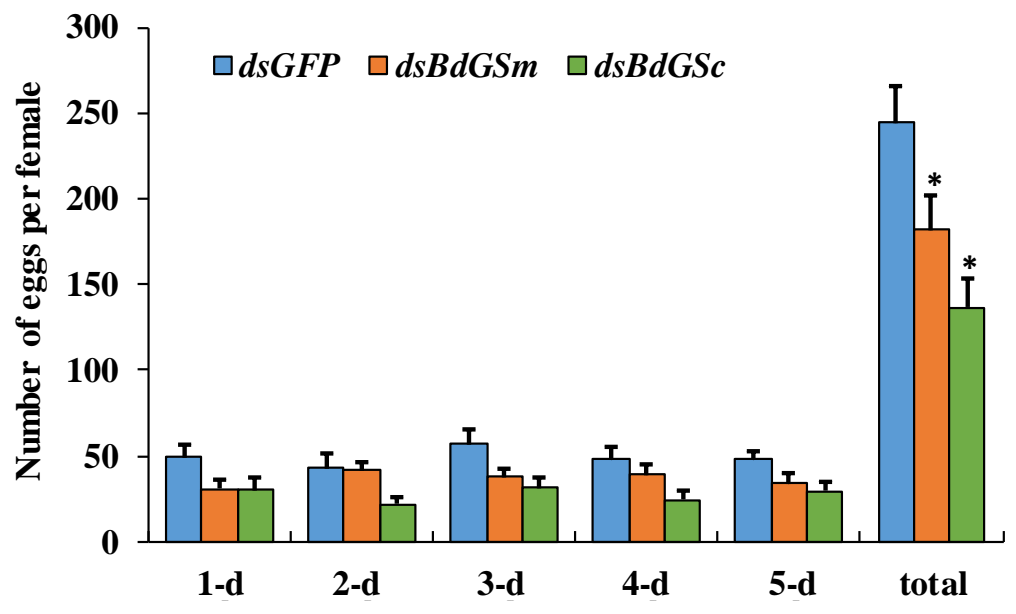

Figure 4. Egg-laying of females when silenced by double-stranded RNA of BdGSs. The bar represents the mean number of eggs $(n=10)$, and the error bar represents the positive standard error of the mean. The asterisk above the bar indicates a significant difference compared with the control as analyzed by Student's $t$-test using SPSS $19.0\left({ }^{*} P<0.05\right)$.

\subsection{MSX Feeding}

When females were fed a high dosage of MSX inhibitor, BdGSm and BdGSc were influenced. The expression of BdGSm was significantly downregulated by a high dosage of MSX, showing a dose-dependent effect (Figure 5A), while the expression of BdGSc was not affected by the MSX inhibitor (Figure 5B). The enzyme determination results showed that the enzyme activity was significantly inhibited at low and high dosages of the inhibitor (Figure 5C). 

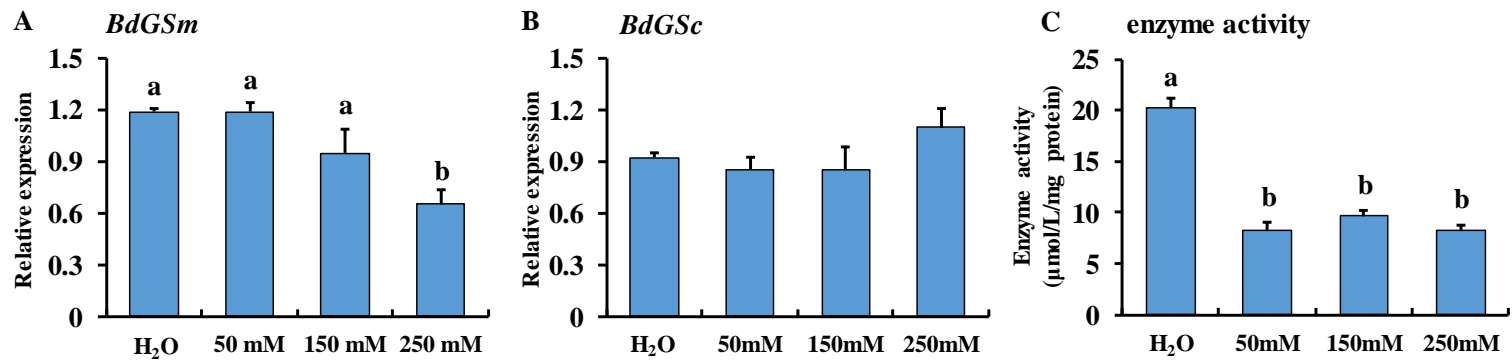

Figure 5. Gene expression of BdGSm (A) BdGSc (B) and enzymatic activity of glutamine synthetase (C) in Bactrocera dorsalis after feeding on the MSX inhibitor. Gene expression was calculated by qBase Plus software. The bar represents the mean gene expression and the error bar represents the positive standard error of the mean. A different letter on the bar indicates a significant difference analyzed by one-way analysis of variance with a Tukey LSD test using SPSS $19.0(P<0.05)$.

To confirm that enzyme inhibition suppresses ovarian development, we dissected the ovaries of females fed on a high dose of MSX and measured their sizes. The ovarian diameter size of the MSX-fed females was significantly smaller than that of the control $(P<0.001$, Figure $6 \mathrm{~A})$. The frequency of each subcategory differed between treatment and control group (Figure 6B). Similar to the RNAi assay, most of the ovaries in the control group were $>1.0 \mathrm{~mm}$, while in the MSX group, most of the ovaries were $<1.0 \mathrm{~mm}$. The gene expression of a vitellogenin ( $B d V g 1)$ and vitellogenin receptor $(B d V g R)$ was detected, and strong suppression of both $B d G S s$ genes was observed in the high-dose MSX treatment group (Figure 6C).
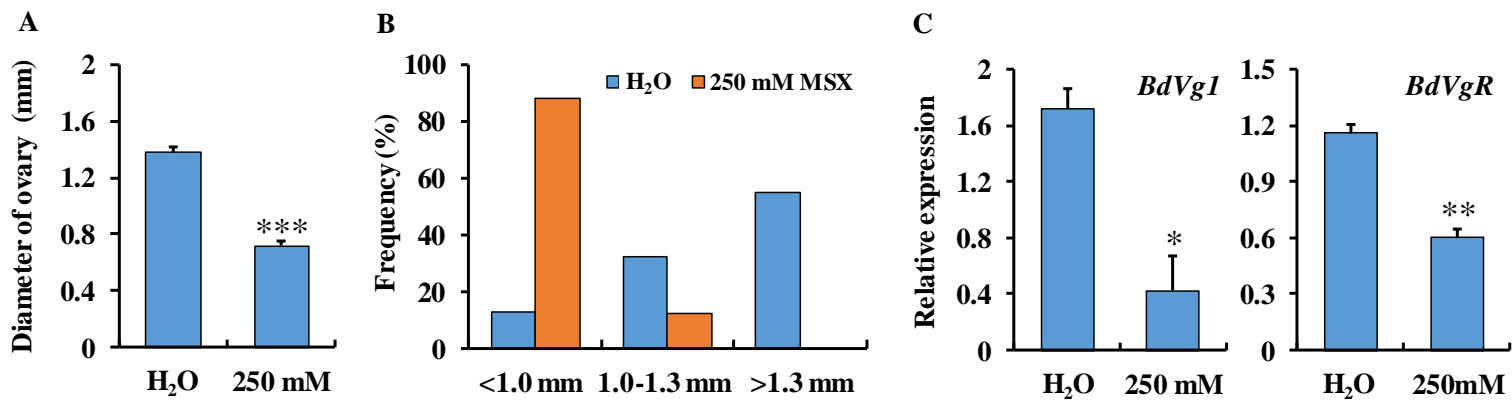

Figure 6. Ovarian diameter $(\mathbf{A})$ and frequency of the ovarian size $(\mathbf{B})$, and the expression of a vitellogenin and its receptor $B d V g R(\mathbf{C})$ in female adults fed a high dose of $250 \mathrm{mM}$ MSX. The bar represents the mean ovarian diameter $(n=20)$, frequency of each ovarian size category, and the mean gene expression, respectively. The error bar indicates the positive standard error of the mean. The expressions of two marker genes were calculated by qBase Plus software. The asterisk on the bar indicates the significant difference analyzed by a Student's $t$-test using SPSS $19.0\left({ }^{*} P<0.05,{ }^{* *} P<0.01,{ }^{* * *} P<0.001\right)$.

\section{Discussion}

Glutamine synthetase converts glutamate and ammonia to glutamine. GS shows negligible glutamine-synthesizing activity in cells at physiological glutamine levels [3], suggesting other roles in insects. In B. dorsalis, GS is involved in the larval development, possibly by regulating ecdysone synthesis [15]. In the present study, we determined the expression of two BdGSs genes during the sexual maturation period of adults, and these genes were widely expressed at different ages of adults. In A. aegypti, GS was also constitutively expressed at all stages [8]. Different isoforms of GSs are expressed in a tissue- and/or development-specific manner which may be involved in distinct biological activities. In $A$. aegypti, the expression increased after a blood meal, indicating active protein synthesis in vitellogenesis [8]. It has been reported that the size of $B$. dorsalis ovary changed greatly during the development [23]. The high expression and enzyme activity of two BdGSs genes may also indicate high protein synthesis in female adults due to vitellogenesis, while the mitochondrial GS is mostly 
involved in energy metabolism. For example, in Drosophila embryo development, mitochondrial GS is highly expressed in the epidermis and in muscle [5].

Isoform differences also result in different tissue-specific expression. For instance, we found that $B d G S m$ was highly expressed in the fat body, while $B d G S c$ was highly expressed in the head and midgut. Tissues of fat body and midgut efficiently incorporate ammonia into amino acids using specific metabolic pathways in A. aegypti [24]. In the fat body, ammonia is first incorporated into the amide side chain of glutamine (Gln) and then into the amino group of glutamic acid (Glu), alanine (Ala), and proline (Pro) by a glutamine synthetase (GS) and glutamate synthase (GltS) pathway (GS/GltS pathway). By contrast, ammonia in the midgut is firstly incorporated into the amino group of Glu and Ala, and then into the amide side chain of Gln. Interestingly, the GS/GltS pathway is not functional in the midgut. GS participates in many biological processes, such as cell growth, energy metabolism, protein and nucleotide synthesis, and immune response [2]. All cells express GS genes but the expression varies according to developmental stage and tissues [8,25]. Similar to BdGSs in $B$. dorsalis, two GSs had different expression patterns in D. melanogaster tissues [5]. Surprisingly, BdGSc was highly expressed in the head of $B$. dorsalis. A high expression of GS has also been found in the neural tissues of $A$. aegypti [26]. This finding suggests a similar neural expression and function in $B$. dorsalis and $A$. aegypti. High expression in neural tissues and the head were also observed in Schistocerca gregaria and Apis cerana [27]. These differences between the two GS isoforms indicate their different roles in insects.

In A. aegypti, the GS provides the glutamine that is essential for the first step of chitin synthesis in the female $A$. aegypti peritrophic matrix [9]. Expression of GS in the gut can be induced by a blood meal [8]. High expression of $B d G S c$ in the midgut may play a similar role in chitin synthesis. In addition to the high expression of $B d G S c$ in larvae, high expression of both $B d G S s$ genes was also observed in adults, especially $B d G S m$ [15], indicating they have critical functions in adult stage. In the present study, we silenced target gene expression using RNAi to suppress both BdGSs genes. This resulted in a smaller female ovary. The significantly decrease of ovarian size indicated a developmental delay though form remained normal. Subsequent egg-laying was also significantly influenced by gene silencing, but the most likely reason was the slowed ovary development. The effect of gene suppression on egg-laying was repeatable in two independent experiments, and a stronger suppression of ovary development was found in the BdGSc treatment.

We inhibited enzyme activity by feeding adults the GS-specific inhibitor MSX. The enzyme activity was significantly inhibited by MSX, but the expression of BdGSm was only inhibited to a low transcriptional level by a high dose of MSX. The mechanism behind the decrease is unclear. MSX-induced glutamine starvation may induce amino-related energy metabolism in the fat body where $B d G S m$ is highly expressed. The limited glutamine was used for basic metabolism, such as nutrition absorption and neurophysiological activity in the gut and head, where BdGSc is highly expressed. Hence, the expression of $B d G S c$ was still at a high level comparable to the control. Similar to $B d G S c$, the GS expression was also not influenced by MSX injection in N. lugens [12]. The fat body is important for reproduction, for example, in vitellogenin synthesis [28]. When we observed the reproductive system of female flies, we found a similar delayed ovarian development. To study the reason for this reproductive inhibition, vitellogenin was used as an index, and it was expressed at a lower transcriptional level. The expression data showed that two BdGSs were differentially expressed in the tested tissues. The mechanisms behind the same downstream role and phenotype may differ in the two target gene RNAi bioassays. BdGSm can regulate vitellogenin synthesis in the fat body directly, while $B d G S c$ can regulate ovarian development by regulating amino acid metabolism in the gut. The regulation of female fecundity by GS was also demonstrated in N. lugens [29], in which RNAi experiments changed the ovarian development of $N$. lugens. The mechanism of GS in regulating female fecundity appears to be complex. Functional analysis showed that a microRNA (miR-4868b) regulates ovarian development by targeting GS in N. lugens [11]. Gene silencing by RNAi also resulted in lower expression of the $V g$ gene in $N$. lugens [29]. A similar lower expression of $B d V g 1$, as well as its receptor 
$B d V g R$, was also found in this study. Other research on GS in N. lugens showed that Gln can activate the target of rapamycin (TOR) signal pathway by promoting the serine/threonine protein kinase AKT and inhibiting 5' AMP-activated protein kinase AMPK phosphorylation activity [12]. These findings demonstrate that GS is an important biomarker in the fecundity of female insects.

\section{Conclusions}

Previous studies indicated that glutamine synthetase (GS) plays a critical role in insects, especially in female fecundity. Two BdGSs are stably expressed in adult $B$. dorsalis in a tissue-specific manner. RNAi and GS-specific inhibitor bioassays delayed ovarian development and lowered egg production in adult female $B$. dorsalis. Both mitochondrial BdGSm and cytoplasmic BdGSc are involved in female fecundity. Considering their important roles in larval metamorphosis, GS genes may be targets for future insect control technologies.

Author Contributions: D.W. and J.J.W. conceived and designed the experiments; M.Y.Z. and S.Y.Z., contributed materials; M.Y.Z. and Y.X.Z. performed the experiments; D.W. and M.Y.Z. analyzed the data; D.W. and J.J.W. wrote the paper; G.S helped draft and revised the manuscript.

Funding: This work was supported in part by the National Key Research and Development Program (2016YFD0200501-9), the Funds from Chongqing Science and Technology Commission (cstc2017shmszx-xdny0031), the PhD Research Funding of Southwest University (SWU118110), and the 111 Project (B18044).

Conflicts of Interest: The authors declare no conflict of interest. The funders had no roles in the design of the study; in the collection, analyses, or interpretation of data; in the writing of the manuscript, or in the decision to publish the results.

\section{References}

1. Lai, X.F.; Gao, H.; Kong, J.; Wang, Q.Y.; Wang, W.J.; Meng, X.H. Cloning and characterization of the glutamine synthetase gene from Chinese shrimp Fenneropenaeus chinensis. Aquacult. Int. 2011, 19, 873-889. [CrossRef]

2. Wang, X.L.; Li, Y.Z.; Yan, Y.; Xu, B.H.; Guo, X.Q. Identification and abiotic stress response of a glutamine synthetase gene (AccGS) from the Asiatic honeybee, Apis cerana cerana (Hymenoptera: Apidae). Eur. J. Entomol. 2014, 111, 1-9. [CrossRef]

3. Eelen, G.; Dubois, C.; Cantelmo, A.R.; Goveia, J.; Bruning, U.; DeRan, M.; Jarugumilli, G.; van Rijssel, J.; Saladino, G.; Comitani, F.; et al. Role of glutamine synthetase in angiogenesis beyond glutamine synthesis. Nature 2018, 561, 63-69. [CrossRef] [PubMed]

4. Kinoshita, S.; Isu, S.; Kaneko, G.; Yamada, H.; Hara, T.; Itoh, Y.; Watabe, S. The occurrence of eukaryotic type III glutamine synthetase in the marine diatom Chaetoceros compressum. Marine Genomics 2009, 2, $103-111$. [CrossRef] [PubMed]

5. Caggese, C.; Barsanti, P.; Viggiano, L.; Bozzetti, M.P.; Caizzi, R. Genetic, molecular and developmental analysis of the glutamine synthetase isozymes of Drosophila melanogaster. Genetica 1994, 94, 275-281. [CrossRef] [PubMed]

6. Caggese, C.; Caizzi, R.; Barsanti, P.; Bozzetti, M.P. Mutations in the glutamine synthetase I ( $g s I$ ) gene produce embryo-lethal female sterility in Drosophila melanogaster. Dev. Genet. 1992, 13, 359-366. [CrossRef] [PubMed]

7. Frenz, L.M.; Glover, D.M. A maternal requirement for glutamine synthetase I for the mitotic cycles of syncytial Drosophila embryos. J. Cell Sci. 1996, 109, 2649-2660.

8. Smartt, C.T.; Kiley, L.M.; Hillyer, J.F.; Dasgupta, R.; Christensen, B.M. Aedes aegypti glutamine synthetase: Expression and gene structure. Gene 2001, 274, 35-45. [CrossRef]

9. Smartt, C.T.; Chiles, J.; Lowenberger, C.; Christensen, B.M. Biochemical analysis of a blood meal-induced Aedes aegypti glutamine synthetase gene. Insect Biochem. Mol. Biol. 1998, 28, 935-945. [CrossRef]

10. Hansen, A.K.; Moran, N.A. Aphid genome expression reveals host-symbiont cooperation in the production of amino acids. Proc. Natl. Acad. Sci. USA 2011, 108, 2849-2854. [CrossRef]

11. Fu, X.; Li, T.; Chen, J.; Dong, Y.; Qiu, J.; Kang, K.; Zhang, W. Functional screen for microRNAs of Nilaparvata lugens reveals that targeting of glutamine synthase by miR-4868b regulates fecundity. J. Insect Physiol. 2015, 83, 22-29. [CrossRef] [PubMed] 
12. Zhai, Y.; Sun, Z.; Zhang, J.; Kang, K.; Chen, J.; Zhang, W. Activation of the TOR signalling pathway by glutamine regulates insect fecundity. Sci. Rep. 2015, 5, 10694. [CrossRef] [PubMed]

13. Clarke, A.R.; Armstrong, K.F.; Carmichael, A.E.; Milne, J.R.; Raghu, S.; Roderick, G.K.; Yeates, D.K. Invasive phytophagous pests arising through a recent tropical evolutionary radiation: The Bactrocera dorsalis complex of fruit flies. Annu. Rev. Entomol. 2005, 50, 293-319. [CrossRef] [PubMed]

14. Wei, D.; Feng, Y.C.; Wei, D.D.; Yuan, G.R.; Dou, W.; Wang, J.J. Female remating inhibition and fitness of Bactrocera dorsalis (Diptera: Tephritidae) associated with male accessory glands. Fla. Entomol. 2015, 98, 52-58. [CrossRef]

15. Zhang, M.Y.; Wei, D.; Li, R.; Jia, H.T.; Liu, Y.W.; Taning, C.N.T.; Wang, J.J.; Smagghe, G. Cytoplasmic glutamine synthetase gene expression regulates larval development in Bactrocera dorsalis (Hendel). Arch. Insect Biochem. Physiol. 2018, 97, e21447. [CrossRef] [PubMed]

16. Wang, J.J.; Wei, D.; Dou, W.; Hu, F.; Liu, W.F.; Wang, J.J. Toxicities and synergistic effects of several insecticides against the oriental fruit fly (Diptera: Tephritidae). J. Econ. Entomol. 2013, 106, 970-978. [CrossRef] [PubMed]

17. Wei, D.; Li, H.M.; Yang, W.J.; Wei, D.D.; Dou, W.; Huang, Y.; Wang, J.J. Transcriptome profiling of the testis reveals genes involved in spermatogenesis and marker discovery in the oriental fruit fly, Bactrocera dorsalis. Insect Mol. Biol. 2015, 24, 41-57. [CrossRef] [PubMed]

18. Wei, D.; Jia, H.T.; Zhang, M.Y.; Li, R.; Smagghe, G.; Wang, J.J. Comparative analysis of differential gene expression profiling of sex-bias fat body of Bactrocera dorsalis (Diptera: Tephritidae) identifying a new vitellogenin gene. Ann. Entomol. Soc. Am. 2018, 111, 43-54. [CrossRef]

19. Shen, G.M.; Jiang, H.B.; Wang, X.N.; Wang, J.J. Evaluation of endogenous references for gene expression profiling in different tissues of the oriental fruit fly Bactrocera dorsalis (Diptera: Tephritidae). BMC Mol. Biol. 2010, 11, 76. [CrossRef]

20. Hellemans, J.; Mortier, G.; De Paepe, A.; Speleman, F.; Vandesompele, J. qBase relative quantification framework and software for management and automated analysis of real-time quantitative PCR data. Genome Biol. 2007, 8, R19. [CrossRef]

21. Livak, K.J.; Schmittgen, T.D. Analysis of relative gene expression data using real-time quantitative PCR and the $2^{-\triangle \Delta C t}$ method. Methods 2001, 25, 402-408. [CrossRef] [PubMed]

22. Cong, L.; Yang, W.J.; Jiang, X.Z.; Niu, J.Z.; Shen, G.M.; Ran, C.; Wang, J.J. The essential role of vitellogenin receptor in ovary development and vitellogenin uptake in Bactrocera dorsalis (Hendel). Int. J. Mol. Sci. 2015, 16, 18368-18383. [CrossRef] [PubMed]

23. Wei, D.; Li, R.; Zhang, M.Y.; Liu, Y.W.; Zhang, Z.; Smagghe, G.; Wang, J.J. Comparative proteomic profiling reveals molecular characteristics associated with oogenesis and oocyte maturation during ovarian development of Bactrocera dorsalis (Hendel). Int. J. Mol. Sci. 2017, 18, 1379. [CrossRef] [PubMed]

24. Scaraffia, P.Y.; Zhang, Q.; Thorson, K.; Wysocki, V.H.; Miesfeld, R.L. Differential ammonia metabolism in Aedes aegypti fat body and midgut tissues. J. Insect Physiol. 2010, 56, 1040-1049. [CrossRef] [PubMed]

25. Youji, H.; Hakvoort, T.B.M.; Vermeulen, J.L.M.; Labruyère, W.T.; D Rudi, D.W.; W Saskia, V.D.H.; Ruijter, J.M.; Uylings, H.B.M.; Lamers, W.H. Glutamine synthetase deficiency in murine astrocytes results in neonatal death. Glia 2010, 58, 741-754.

26. Avisar, N.; Shiftan, L.; Ben-Dror, I.; Havazelet, N.; Vardimon, L. A silencer element in the regulatory region of glutamine synthetase controls cell type-specific repression of gene induction by glucocorticoids. J. Biol. Chem. 1999, 274, 11399-11407. [CrossRef]

27. Boyan, G.; Williams, L. Embryonic development of the insect central complex: insights from lineages in the grasshopper and Drosophila. Arthropod Struct. Dev. 2011, 40, 334-348. [CrossRef] [PubMed]

28. Chen, S.L.; Lin, C.P.; Lu, K.H. cDNA isolation, expression, and hormonal regulation of yolk protein genes in the oriental fruit fly, Bactrocera dorsalis (Hendel) (Diptera: Tephritidae). J. Insect Physiol. 2012, 58, 763-770. [CrossRef]

29. Zhai, Y.; Zhang, J.; Sun, Z.; Dong, X.; He, Y.; Kang, K.; Liu, Z.; Zhang, W. Proteomic and transcriptomic analyses of fecundity in the brown planthopper Nilaparvata lugens (Stål). J. Proteome Res. 2013, 12, 5199-5212. [CrossRef]

(C) 2019 by the authors. Licensee MDPI, Basel, Switzerland. This article is an open access article distributed under the terms and conditions of the Creative Commons Attribution (CC BY) license (http://creativecommons.org/licenses/by/4.0/). 